\title{
Preface to TCHES Volume 2021
}

\author{
Elke De Mulder ${ }^{1}$ and Peter Schwabe ${ }^{2,3}$ \\ 1 Rambus Cryptography Research \\ 4453 North First Street, Suite 100 \\ San Jose, CA 95134 \\ USA \\ edemulder@rambus.com \\ ${ }^{2}$ Max Planck Institute for Security and Privacy \\ Universitätsstraße 140 \\ 44799 Bochum \\ Germany \\ ${ }^{3}$ Radboud University \\ Toernooiveld 212 \\ 6525 EC Nijmegen \\ The Netherlands \\ peter@cryptojedi.org
}

Having been established in 1999, the Cryptographic Hardware and Embedded Systems (CHES) conference is today the premier venue for research on both design and analysis of cryptographic hardware and software implementations. As an area conference of the International Association for Cryptologic Research (IACR), CHES bridges the cryptographic research and engineering communities, and attracts participants from academia, industry, government and beyond.

CHES 2021 was held as a virtual event on September 13-17, 2021 after careful discussion half a year prior to the conference dates about whether or not it would be realistic to hold an in-person event due to the ongoing COVID-19 pandemic. It was the second exclusively virtual and the twenty-third overall edition of the CHES conference.

Since 2018, CHES is run under a hybrid model as a mixture of journal publications and conference presentations. The papers constituting the CHES 2021 program were published in the IACR Transactions on Cryptographic Hardware and Embedded Systems (TCHES) Volume 2021, Issues 1, 2, 3 and 4 under a platinum open-access model. The journal is published by Ruhr-Universität Bochum; the managing director and publishing editor is Tim Güneysu.

This is the first year HotCRP was adopted as submission and review system. Despite some learning mistakes and per-deadline adjustments due to a still-evolving platform, the experiment was rather successful. One major feature still lacking from these review systems is the ability to deal with resubmissions and the administrative overhead it creates for the program chairs to keep track of previous reviewers and discussions.

To help the rebuttal process and aid the authors in deciding what to focus on while preparing their answer, we opted to include a section in the review form where reviewers could ask questions they deemed most important, with the goal to receive answers in the rebuttal.

Since it was an all-virtual and world-wide event, the conference presentations themselves were limited to five minutes; the full twenty-minute presentations were pre-recorded and made available online for people to enjoy at their own leisure. 
Table 1: Submission statistics of TCHES Volume 2021.

\begin{tabular}{|l|c|c|c|c|}
\hline & Issue 1 & Issue 2 & Issue 3 & Issue 4 \\
\hline Number of new submissions & 45 & 44 & 41 & 62 \\
\hline Number of major revisions from previous issues & 4 & 7 & 24 & 10 \\
\hline Number of re-submissions from previous issues & 3 & 1 & 4 & 5 \\
\hline Number of submissions (total) & 52 & 52 & 69 & 77 \\
\hline Number of accepted submissions (total) & 17 & 13 & 26 & 22 \\
\hline Number of accepted submissions (new only) & 13 & 7 & 3 & 11 \\
\hline Acceptance rate & $32.6 \%$ & $25 \%$ & $37.6 \%$ & $28.5 \%$ \\
\hline
\end{tabular}

The submission statistics of TCHES Volume 2021 are summarized in Table 1. The four issues have received a total of 192 new submissions; counting the resubmissions, a total of 250 submissions were reviewed for this volume. Out of those 78 have been accepted, making a global acceptance rate of $31 \%$. The higher acceptance rate is mostly due to the process of major revisions.

After voting, the Editorial Board conferred the CHES 2021 best paper award to My other car is your car: Compromising the Tesla Model X keyless entry system by Lennert Wouters, Benedikt Gierlichs and Bart Preneel. The program included two invited talks: From CHERI to Arm Morello: Architectural Support for Memory Protection and Software Compartmentalization by Robert Watson (University of Cambridge) and Hardware for privacy engineering by Carmela Troncoso (EPFL).

New this year was the addition of an optional artifact review and archiving process for accepted papers which was deftly chaired and organized by Douglas Stebila from the University of Waterloo. The main goal was to to ensure functionality and reusability of artifacts. All about this process can be read in a separate preface. We sincerely hope that this evaluation of artifacts will continue to be part of the TCHES/CHES publication model.

Acknowledgments. Creating a journal and conference is a labor-intensive task and a lot of minds and hands have to come together and put in effort to make it happen. We would like to thank all the parties involved. We would like to thank our sponsors for their generous contribution: Rambus, CryptoExperts, NXP, SciEngines, Infineon, Open Security Research and Qualcomm. We would like to extend thanks to the general chairs Liji Wu, Guoqiang Bai, Zhe Liu, and Junfeng Fan. A special shout-out goes to Douglas Stebila who took on the daunting task to organize the first ever CHES artifact review process. Two hundred fifty submissions, spread over four deadlines resulted in a big workload. The result achieved could not have been possible without the dedication and professionalism of the Editorial Board members and the external reviewers. Without the help of Kevin McCurley and Kay McKelly who administer and maintain not only the HotCRP software, but take care of all the technical aspects of running a virtual conference, neither the journal, nor the conference would have happened. The publication of TCHES was supported by Tim Güneysu in the role of Managing Editor and Markus Krausz for the practical issues; constituent papers all used a $\mathrm{LT}_{\mathrm{E}} \mathrm{X}$ style originally authored by Gaëtan Leurent. A special thanks also goes to the CHES Steering Committee for support and advice during these unprecedented time and the hard decision to once more have a virtual-only conference. Last, but not least we are indebted to the authors of all the submissions. Without those, there would be no CHES in the first place.

September 2021

Elke De Mulder Peter Schwabe 


\section{Editorial Board}

Diego F. Aranha

Manuel Barbosa

Sonia Belaïd

Benjamin Beurdouche

Begül Bilgin

Billy Bob Brumley

Chris Brzuska

Ileana Buhan

Rajat Subhra Chakraborty

Tung Chou

Chitchanok Chuengsatiansup

Jeroen Delvaux

François Dupressoir

Stefan Dziembowski

Barış Ege

Fatemeh Ganji

Daniel Genkin

Benedikt Gierlichs

Dahmun Goudarzi

Hannes Gross

Dong-Guk Han

Annelie Heuser

Xiaolu Hou

Andreas Hülsing

Elif Bilge Kavun

Boris Köpf

Kerstin Lemke-Rust

Tancrède Lepoint

Patrick Longa

Julio López

Marco Macchetti

Stefan Mangard

Nele Mentens

Elke De Mulder

Ruben Niederhagen

David Oswald

Colin O'Flynn

Daniel Page

Peter Pessl

Stjepan Picek

Thomas Pornin

Thomas Pöppelmann

Francesco Regazzoni

Francisco Rodríguez-Henríquez

Pascal Sasdrich

Kazuo Sakiyama

Tobias Schneider
Aarhus University, Denmark

University of Porto (FCUP)

\& INESC TEC, Portugal

CryptoExperts, France

Mozilla, France

Rambus Cryptography Research, The Netherlands

Tampere University, Finland

Aalto University, Finland

Riscure B.V., The Netherlands

IIT Kharagpur, India

Academia Sinica, Taiwan

The University of Adelaide, Australia

Open Security Research, China

University of Bristol, UK

University of Warsaw, Poland

Riscure B.V., The Netherlands

Worcester Polytechnic Institute, USA

University of Michigan, USA

KU Leuven, Belgium

Independent Researcher, FR

SGS Digital Trust Services, Austria

Kookmin University, South Korea

Université de Rennes, Inria, CNRS, IRISA, France

Slovak University of Technology in Bratislava, Slovakia

Eindhoven University of Technology, The Netherlands

University of Passau, DE

Microsoft Research, UK

Bonn-Rhein-Sieg University of Applied Sciences, Germany

Google, USA

Microsoft Research, USA

University of Campinas, Brazil

Kudelski Group, Switzerland

Graz University of Technology, Austria

Leiden University, The Netherlands

\& KU Leuven, Belgium

Rambus Cryptography Research, USA

University of Southern Denmark, Denmark

The University of Birmingham, UK

Dalhousie University, Canada

University of Bristol, UK

Infineon Technologies, Germany

TU Delft, The Netherlands

NCC Group, Canada

Infineon Technologies, Germany

University of Amsterdam, The Netherlands

\& ALaRI - USI, Switzerland

CINVESTAV, Mexico

Ruhr University Bochum, Germany

The University of Electro-Communications, Japan

NXP Semiconductors, Austria 
Peter Schwabe

Martijn Stam

Marc Stöttinger

Takeshi Sugawara

Petr Svenda

Adrian Thillard

Mehdi Tibouchi

Yosuke Todo

Gilles Van Assche

Srinivas Vivek

Christine van Vredendaal

Bo-Yin Yang

Bohan Yang

Yuval Yarom

\section{External Reviewers}

\author{
Ali Abbasi \\ Estuardo Alpírez Bock \\ Gustavo Banegas \\ Xavier Bonnetain \\ Olivier Bronchain \\ Fabio Campos \\ Łukasz Chmielewski \\ Ibrahima Diop \\ Nisrine Jafri \\ Damien Marion \\ Shyam Murthy \\ Sioli O'Connell \\ Robert Primas \\ Martin Rehberg \\ Tania Richmond \\ Mélissa Rossi \\ Ahmad-Reza Sadeghi \\ Victor Servant \\ Florian Unterstein \\ Praveen Vadnala \\ Annapurna Valiveti \\ Fernando Virdia \\ Brecht Wyseur
}

Max Planck Institute for Security and Privacy, Germany

\& Radboud University, The Netherlands

Simula UiB, Norway

Hessen3C, Germany

The University of Electro-Communications, Japan

Masaryk University, Czech Republic

Ledger, France

NTT Corporation, Japan

NTT Corporation, Japan

STMicroelectronics, Belgium

IIIT Bangalore, India

NXP Semiconductors, The Netherlands

Academia Sinica, Taiwan

Tsinghua University, China

The University of Adelaide

\& Data61, Australia 